\title{
The Westerbork Flexible Filterbank Backend
}

\author{
Marco Kouwenhoven \\ Astronomical Institute, Utrecht University \\ Lodie Voûte, Michiel van der Klis \\ Astronomical Institute, University of Amsterdam \\ Hans Weggemans, Will Deich \\ Netherlands Foundation for Research in Astronomy, Dwingeloo \\ Gautam Vasisht, Shri Kulkarni \\ California Institute of Technology
}

\section{Introduction}

The Westerbork Synthesis Radio Telescope (WSRT) has recently been fitted with a new backend designed for pulsar detection. This backend is a filterbank with 32 channels, for both the $\mathrm{X}$ and $\mathrm{Y}$ polarised signals. A novel feature is that the channel bandwidths are digitally tunable between $6.25 \mathrm{kHz}$ and 2.8 $\mathrm{MHz}$ (Vasisht et al. in preparation). The time constant of the integration which follows detection can be digitally set at values between circa $50 \mathrm{~ms}$ and $10 \mu \mathrm{s}$. For each observing frequency and each pulsar (with a known dispersion measure) we can choose an optimal bandwidth and signal smoothing.

\section{The Filterbank}

The backend has been constructed by Shri Kulkarni's group at Caltech and was installed by Lodie Voûte and Hans Weggemans in Westerbork. The software was adapted and extended by Will Deich. It has been fully operational since the end of August 1995.

For detecting pulsars the signals of all 14 Westerbork dishes are added coherently and split into 8 frequency bands for both polarizations. Each band is mixed with 4 (computer controlled) local oscillator signals and put into 64 digital tunable filters. Then the signals are squared and the DC-components are removed. After smoothing they are 2-bit sampled and stored on disk or tape. The smoothing filter and the sampling interval again are computer controlled and are set in such a way that the signal is sampled almost at Nyquist rate.

The small effective beam of the Westerbork telescope in the tied-array mode make it very inefficient to do untargeted searches. But combining the imaging and polarization capabilities of the telescope with the timing capabilities of this backend we can do efficient targeted searches. 
Table 1. Parameters of the Westerbork Flexible Filterbank Backend

\begin{tabular}{|ll|}
\hline Number of channels & 32 (future: 8000 ) \\
Number of polarizations & 2 (future: 4 ) \\
Bandwidth per channel & $6.25 \ldots 200 \mathrm{kHz}$ (in steps) \\
& $0.35 \ldots 2.8 \mathrm{MHz}$ (continous) \\
Smoothing filter & $24 \mathrm{~Hz} \ldots 100 \mathrm{kHz}$ (in steps) \\
Sampling interval & $51.2,102.4,204.7 \mu \mathrm{s} \ldots$ \\
& (future: $0.4 \mu \mathrm{s} \ldots$ ) \\
DC-removal time constant & $2 \ldots 100 \mathrm{~s}$ (in steps) \\
\hline
\end{tabular}

\section{Projects}

At this moment we are planning the following projects:

- Pulse search towards steep spectrum sources discovered in the Westerbork Northern Sky Survey (WENSS) and the VLA Faint Images of the Radio Sky at Twenty centimeter (FIRST).

- Pulse search towards highly polarized objects in the WENSS.

- Pulsar search in a few globular clusters.

- Pulse search towards radio sources near possible supernova remnants in the WENSS.

- Timing of a few northern sky millisecond pulsars.

- Investigation of giant pulses.

\section{Future}

At this moment we are working on further improvements of the machine:

- Implementation of a faster workstation: maximum sampling rate: 2.5 $\mathrm{MHz}$.

- Detection of all 4 Stokes parameters.

- Extension of the number of channels to 8000 .

Acknowledgments. I would like to thank the Leids Kerkhoven-Bosscha Fonds for supplying travel money for visiting this conference. This research is supported by NWO under grant PGS 78-277. 